\title{
Asymptotic form at large $r$ of a third-order linear homogeneous differential equation for the ground-state electron density of the He atom
}

\author{
N. H. March, ${ }^{1,2}$ Á. Nagy, ${ }^{3}$ and C. Amovilli ${ }^{4}$ \\ ${ }^{1}$ Department of Physics, University of Antwerp, Groenenborgerlaan 171, B-2020 Antwerp, Belgium \\ ${ }^{2}$ Oxford University, Oxford, England \\ ${ }^{3}$ Department of Theoretical Physics, University of Debrecen, H-4010 Debrecen, Hungary \\ ${ }^{4}$ Dipartimento di Chimica e Chimica Industriale, Università di Pisa, Via Risorgimento 35, 56126 Pisa, Italy
}

(Received 22 November 2007; published 6 March 2008)

\begin{abstract}
In earlier work a linear differential equation satified by the Schwartz ground-state electron density $\varrho(r)$ for (non-relativistic) He-like atomic ions with large atomic number $Z$ has been derived. Here, we utilize the asymptotic expansion at large $r$ given by Amovilli and March for the neutral He atom. We thereby show that a linear differential equation of the same general shape as that satisfied by the Schwartz $\varrho(r)$ again emerges for the neutral $\mathrm{He}$ atom itself, in the asymptotic limit of large $r$. We argue that essential input into the final differential equation for the $\mathrm{He}$ ground-state electron density will be the ionization potential plus the atomic polarizability.
\end{abstract}

DOI: 10.1103/PhysRevA.77.034501

PACS number(s): 31.15.-p

Going back at least to the early work of HoffmannOstenhof and Hoffmann-Ostenhof [1], it is known that the ground-state density $\varrho(r)$ of spherical atoms (e.g., $\mathrm{Ne}$ and Ar) falls off at sufficiently large $r$ as

$$
\varrho(r) \approx N r^{n} \exp (-2 \sqrt{2 I} r), \quad r \rightarrow \infty,
$$

where $I$ is the ionization potential in atomic units.

Here we shall be concerned with the two-electron $\mathrm{He}$ atom ground-state density $\varrho(r)$ and, in particular, with the ongoing search for the differential equation this $\varrho(r)$ satisfies. A first step toward this objective was taken by Gál, March, and Nagy [2]. These authors adopted as their starting point the study of Schwartz [3] who considered (always via the nonrelativistic Schrödinger equation, however) the large atomic number $Z$ limit of two-electron He-like ions of nuclear charge $\mathrm{Ze}$. His result had the form

$$
\varrho(r)=\frac{2 Z^{3}}{\pi}\left[1+\frac{2}{Z} \chi(r)\right] \exp (-2 Z r)
$$

where $\chi(r)$ was calculated explicitly. The major finding in [2] was that $\varrho(r)$ in Eq. (2) satisfied exactly a third-order linear homogeneous differential equation [see Eq. (3) below].

The motivation for reopening the search for such an exact differential equation for the neutral $\mathrm{He}$ atom itself, with $Z=2$, comes from the very recent study by two of us [4] of the long-range asymptotic behavior of $\varrho(r)$ in this case. In particular, we take as the starting point of the present study the asymptotic, large $r$ expansion given in Eqs. (31)-(33) of $[4]$.

We show below that the Amovilli-March (AM) large $r$ form satisfies indeed a third-order linear homogeneous differential equation having precisely the same shape, but of course differs in fine details. As in [2] the form is explicitly

$$
P_{3}(r) \varrho^{\prime \prime \prime}(r)+P_{2}(r) \varrho^{\prime \prime}(r)+P_{1}(r) \varrho^{\prime}(r)+P_{0}(r) \varrho(r)=0 .
$$

Treating the AM asymptotic form as exact, one is then led (see below) to the fact that the $P_{i}(r)$ in Eq. (3) are polynomials all with the highest power of $r^{3}$. Of course, even if this proves the correct structure of any final differential equation for $\varrho(r)$ for the He itself, the coefficients of the low-order terms will be changed.

Below we present an outline of the derivation of the form (3) from the AM asymptotic large $r$ expansion of $\varrho(r)$ [4] [see especially Eqs. (31)-(34)]. In particular, Eq. (31) yields, at large $r$,

$$
\exp (2 \sqrt{2} I r) \varrho(r)=g(r)=A r^{k}\left[1+\frac{C_{1}}{r}+\frac{C_{2}}{r^{2}}+\frac{C_{3}}{r^{3}}+O\left(\frac{1}{r^{4}}\right)\right] .
$$

Differentiating Eq. (4) three times then leads to the form

$$
\varrho g^{\prime \prime \prime}=g\left[\varrho^{\prime \prime \prime}+6 \sqrt{2 I} \varrho^{\prime \prime}+24 I \varrho^{\prime}+16 I \sqrt{2 I} \varrho\right] \text {. }
$$

Using expression (4) for $g$ and truncating the expansion at $r^{-3}$ we find the general form of Eq. (3), where the polynomials have the form

$$
\begin{gathered}
P_{3}(r)=C_{3}+C_{2} r+C_{1} r^{2}+r^{3}, \\
P_{2}(r)=6 \sqrt{2 I}\left[C_{3}+C_{2} r+C_{1} r^{2}+r^{3}\right], \\
P_{1}(r)=24 I\left[C_{3}+C_{2} r+C_{1} r^{2}+r^{3}\right],
\end{gathered}
$$

and

$$
\begin{aligned}
P_{0}(r)= & {\left[16 I \sqrt{2 I} C_{3}-k(k-1)(k-2)+16 I \sqrt{2 I} C_{2} r\right.} \\
& \left.+16 I \sqrt{2 I} C_{1} r^{2}+16 I \sqrt{2 I} r^{3}\right] .
\end{aligned}
$$

Comparing Eq. (4) with Eq. (31) of [4], namely, 


$$
\sqrt{\varrho(r)} \approx M r^{\beta-1}\left[1+\frac{A_{1}}{r}+\frac{A_{2}}{r^{2}}+\frac{A_{3}}{r^{3}}\right] \exp (-\sqrt{2 I} r),
$$

we obtain

$$
\begin{gathered}
C_{1}=2 A_{1}, \\
C_{2}=A_{1}^{2}+2 A_{2},
\end{gathered}
$$

and

$$
C_{3}=2\left(A_{3}+A_{1} A_{2}\right) .
$$

It was shown in [4] that for $\mathrm{He}$

$$
\begin{gathered}
A_{1}=\frac{\beta(\beta-1)}{2[\sqrt{2 I}(\beta-1)-1]}, \\
A_{2}=\frac{A_{1}(\beta-1)(\beta-2)}{2[\sqrt{2 I}(\beta-2)-1]}, \\
A_{3}=\frac{A_{2}(\beta-2)(\beta-3)}{2[\sqrt{2 I}(\beta-3)-1]}+\frac{\alpha}{2[\sqrt{2 I}(\beta-3)-1]},
\end{gathered}
$$

and

$$
\beta=\frac{k}{2}+1=\frac{1}{\sqrt{2 I}} .
$$

Of course, while we expect the highest powers of $r$ in polynomials $P_{i}(r)$ entering Eq. (3) will be derivable from the result (32) and (33) in [4], we shall not pursue the details further.

What we emphasize here is that such a differential equation as in Eq. (6) will then be characterized by (i) the ionization potential $I$ and (ii) the polarizability $\alpha$, which enters the AM coefficient $A_{3}$ and, consequently, $C_{3}$ occurring in Eq. (4).

For the future, should it prove, as we conjecture, that the shape (3) is formally exact for the ground-state electron density of the $\mathrm{He}$ atom, then the small $r$ expansion of $\varrho(r)$ should also be invoked to fix the low-order terms in the polynomials $P_{i}(r)$ entering Eq. (6). In fact, from the study of Nagy and Sen [5], one has a relation between derivatives of $\varrho(r)$ at the origin of the form

$$
\bar{\varrho}^{\prime \prime \prime}(0)=Z\left[-5 \bar{\varrho}^{\prime \prime}(0)+12 Z^{2} \bar{\varrho}(0)+4 \eta_{1}(0)\right] .
$$

The spherical average of the density can be written as

$$
\bar{\varrho}(r)=\eta_{0}(r)+\sum_{l>0} r^{2 l} \eta_{l}(r)
$$

The last term in Eq. (18) contains not the density itself, but only a part of the density: the part corresponding to $l=1$; that is, there is a contribution only from the " $p$ electron density" [5]. In this aspect Eq. (18) differs from Eq. (3) that contains only the density and its derivatives.

In summary, the shape (3) of the linear third-order homogeneous differential equation satisfied by the Schwartz limiting density (2) for He-like atomic ions with large atomic number $Z$ also follows from the AM asymptotic large $r$ expansion for the ground state of the He atom itself. Then the $\mathrm{AM}$ result indicates that such a differential equation will be characterized by the ionization potential $I$, which should occasion no surprise due to the exact asymptotic result quoted in Eq. (1), and also by the atomic polarizability $\alpha$. The origin of the dependence on $\alpha$ can be traced back, through density functional theory, to the large $r$ form of the exchangecorrelation potential $V_{x c}$ as $-e^{2} / r-\alpha / 2 r^{4}[6,7]$.

N.H.M. acknowledges that his contribution to the present study began during a visit to the Physics Department, University of Antwerp. He thanks Professor D. Van Dyck and Professor D. Lamoen for support for his continuing affiliation. N.H.M. brought his contribution to this work to fruition during a visit to the University of Debrecen. He wishes to thank Professor Á. Nagy for very generous hospitality. C.A. and Á.N. acknowledge the Bilateral Scientific Cooperation between Italy and Hungary sponsored by Consiglio Nazionale delle Ricerche and the Hungarian Academy of Sciences. Á.N. acknowledges OTKA Grant No. T67923.
[1] M. Hoffmann-Ostenhof and T. Hoffmann-Ostenhof, Phys. Rev. A 16, 1782 (1977).

[2] T. Gál, N. H. March, and Á. Nagy, Chem. Phys. Lett. 305, 429 (1999).

[3] C. Schwartz, Ann. Phys. (N.Y.) 2, 156 (1959).
[4] C. Amovilli and N. H. March, J. Phys. A 39, 7349 (2006).

[5] Á. Nagy and K. D. Sen, J. Chem. Phys. 115, 6300 (2001).

[6] C. O. Almbladh and U. von Barth, Phys. Rev. B 31, 3231 (1985).

[7] N. H. March, Phys. Rev. A 65, 034501 (2002). 